bairn and Glober $(3,117 ; 1970)$. They found that twelve women out of a group of thirty who had suffered postoperative thromboembolism had been using oral contraceptives during the month before surgery, while only nine out of a group of sixty carefully matched controls had been doing so. From this they estimate that the risk is almost four times greater for women taking the pill than for those who are not, but Vessey et al. are careful to point out that the numbers involved are so small that the estimate is necessarily rough.

A causal relationship between the use of oral contraceptives and the occurrence of thromboembolism in previously healthy women has already been established by four retrospective studies; and according to Vessey et al. the findings of the latest study are in broad agreement with these earlier results. Although the numbers involved were too small to allow an analysis of the effects of different types of contraceptive, the Oxford team say that the pills containing a high concentration of oestrogen seem to be the most hazardous and that the risk is unrelated to the time during which the pills have been taken.

Fortunately, as this and the earlier studies indicate, the extra risk of thromboembolism due to the pill declines rapidly after the pill is discontinued, so that if a woman is advised to give up the pill before an operation, a month is long enough to ensure that the extra risk disappears.

While the results from this and other studies probably apply to women from most of the developed countries, Dr M. Potts of the International Planned Parenthood Federation points out that they do not necessarily apply to women from underdeveloped countries where the incidence of thrombosis is much lower; the risks involved in taking the pill may well be less for these women.

\section{RIBOSOMAL RNA}

\section{Maturation in a Missing Link}

from our Cell Biology Correspondent

Recently (Nature, 22\%, 338; 1970) I mentioned that the maturation of ribosomal RNAs in prokaryotes and eukaryotes has an outstanding common feature; the process seems to involve the cleavage of precursor molecules larger than the mature ribosomal RNAs. But there are differences; in eukaryotes the two riboosmal RNAs are produced by splitting a single large precursor molecule whereas in bacteria the two ribosomal RNAs are derived from two discrete precursors each only slightly larger than the mature species. In the light of this distinction between prokaryotes and eukaryotes, it would be of considerable interest to know how ribosomal RNA is synthesized and processed in the dinoflagellates, organisms which are seldom in the limelight but which, although classified as eukaryotes, have retained several features characteristic of the prokaryotes.

The dinoflagellates, for example, seem to lack the basic chromosomal proteins characteristic of fully fledged eukaryotes and their chromosomes, or more precisely their DNA-containing bodies, are reminiscent of bacterial nucleoids. Moreover, the chromatids in dinoflagellates segregate by attaching to the nuclear envelope, which extends and effects separation, rather than to a mitotic spindle. And the structure of the nuclear envelope itself varies from that typical of eukaryotes to a bilamellar structure with unusual vesicles believed to be involved in transport from nucleus to cytoplasm.

With so many features characteristic of prokaryotes there was a distinct possibility that processing of ribosomal RNA in the dinoflagellates might resemble that in the bacteria. Rae (J. Cell. Biol., 46, 106; 1970), however, has shown recently that this is not the case. In Gyrodinium cohnii, at least, the $16 \mathrm{~S}$ and $25 \mathrm{~S}$ ribosomal RNAs, which have a G plus $C$ content of 46 per cent, are derived from a single large precursor molecule sedimenting at $38 \mathrm{~S}$. This is apparently cleaved to yield a $16 \mathrm{~S}$ piece and a $27 \mathrm{~S}$ piece which is subsequently converted to the mature $25 \mathrm{~S}$ species. Moreover, the nucleoli in $G$. cohnii have the typical structure with a central filamentous region surrounded by a peripheral granular zone. And even in the more primitive Prorocentrum and Exuviella, R. Guillard, working at Woods Hole, has apparently seen in the electron microscope typical nucleoli. At least as far as ribosomal RNA is concerned the dinoflagellates are respectable eukaryotes.

To what extent their ribosomal RNA is methylated remains to be seen, but the significance of methylated bases in ribosomal and tRNAs even in $E$. coli, let alone the dinoflagellates, is obscure. The outstanding difficulty in tackling the problem has, of course, been isolating mutants which contain under-methylated RNAs. For $E$. coli that has now been overcome by Björk and Isaksson (J. Mol. Biol., 51, 83; 1970), who have devised a screening procedure for mutants with RNA which has the capacity to accept methyl groups. of ten such mutants, six have under-methylated tRNAs and one under-methylated ribosomal RNA as well. The tRNA mutants are deficient in the enzyme(s) responsible for methylating the 5 position of uracil, while the one double mutant lacks this enzyme and the enzyme responsible for producing 1-methylguanine in ribosomal RNA. The complete absence of 5-methyluracil in tRNA and 1-methylguanine in ribosomal RNA is not lethal and the properties of the mutants suggest different enzymes are responsible for the methylation of the different RNAs. With luck, further characterization of these and other similar mutants may explain the significance of methylated bases in the translation machinery.

\section{How to Gel Recruils}

\section{from a Correspondent}

Fisheries biologists have often assumed that the number of young entering a fish stock each year was independent of the quantity of the parent stock. The assumption was reasonable because this process of recruitment is very variable. But in the past decade the recruitment to two fish stocks important to British fishermen-the East Anglian herring fishery and the Barents Sea cod fishery-has declined during a period of heavy fishing and so the assumption was questioned. The experts met to discuss the relationship between stock and recruitment at a symposium held from July 7 to 10 at Aarhus, in Denmark, under the auspices of the International Council for the Exploration of the Sea, the International Commission for Northwest Atlantic Fisheries and the Food and Agriculture 